\title{
Blade loss studies in low-pressure turbines - from blade containment to controlled blade-shedding
}

\author{
R. Ortiz ${ }^{1}$, M. Herran ${ }^{2,3}$ \& H. Chalons ${ }^{2}$ \\ ${ }^{I}$ Onera, DADS/CRD, Structural and Dynamic Strength Unit, \\ Lille, France \\ ${ }^{2}$ Turbomeca-Safran Group, Methods and Development Tools for \\ Mechanical Design and Analysis, Bordes, France \\ ${ }^{3}$ Université de Lyon, CNRS, Insa-Lyon, LaMCoS, Villeurbanne, France
}

\begin{abstract}
Activities performed in the "Structural Design and Dynamic Strength" research unit of ONERA-Lille mainly aim at analysing the behaviour of aeronautical structures under dynamic transient loadings (crash, impact, explosion...). In this frame, recent studies are performed in collaboration with TURBOMECA (French turbine manufacturer) to evaluate and develop numerical methodologies for the analysis of "blade-shedding" events in low-pressure turbines.

Blade-shedding is part of a safety process to preserve turbines disks from burst in the event of over-speed regime and consists in generating controlled blades ruptures in order to decrease centrifugal efforts sustained by the disks. Such a procedure however leads, on the one hand, to impacts from the released blades onto the turbine rings and containment shields and, on the other hand, to unbalance transient centrifugal efforts in the turbine, which create severe loads at the engine mounting components and bolted assemblies.

Keywords: blade-off, low pressure turbine, Finite Element, Explicit code, blade shedding.
\end{abstract}

\section{Introduction}

These engine mounting loads constitute dimensioning parameters for the engine structure and are estimated with a whole engine test. The proposed works 
therefore consist in evaluating the capacity of Finite Element (FE) explicit methodologies to handle with such a dynamic event, with specific emphasis laid upon the understanding of blade rupture process, up to the impact with the containment shield, and the prediction of the efforts supported by the engine mounting components. The ultimate objective should be to contribute to the reduction of the turbine design phase duration and costs (including the suppression or the simplification of certification engine test).

Already, the performed simulations permitted to identify some needs in terms of model improvements to better predict numerically, then pass the bladeshedding loads to the engine mounting points, which represent a key-point in turbine certification process.

A full-scale 3D FE model of a recent turbine and structural environment (protection panel, engine mounting component...) has then been developed with the Europlexus code for feasibility purposes: the blades trajectory after rupture, their impact/penetration through the turbine rings and stopping by the containment shield can now be studied

\section{State of the art}

The prediction of the engine mountings loads using finite element method requires a special study of mechanical phenomena occurring in the "blade shedding" phase and to simulate the engine behaviour. On the literature, the problem has no equivalence because Turbomeca is the first manufacturer which uses this technology and so the nearest problem is the blade out on the free turbine. The blade out is defined by the release of one blade at the maximum defined rotational speed. The mechanical problem is then to demonstrate that no high-energy debris is ejected from the engine and the deceleration of the unbalanced rotor, due to the blade loss, does not lead to hazardous behaviour of the engine. For example, Sun [1] has recently simulated the transient rotor dynamics due the loss of one blade and investigated the effect of thermal growths for the ball bearing

First, the Blade Shedding engine test has been analysed in order to understand the scenario of the phenomena. The result of the analysis [2] gives a global idea of the different sequences of the "Blade Shedding": the release of all the blades takes a few ms while high vibration levels in the structure and loads on the mounting are observed during a few tenths of seconds.

Nevertheless the sequence of the release of the blade has not been identified and remains one of the most unknown of the problem.

Indeed, the transient rotor dynamics needs to be simulated. By the past, Guilhen et al. [3] has proposed a Newmark Algorithm with Alpha=1/2 and Beta $=1 / 2$ in order to solve the problem and a modal reduction in order to save time calculation. In a second time, a recent work [4] has been achieved to modelize the transient rotor dynamic with beam element on the Europlexus finite element explicit code and allows, on the one hand, simulate the mountings loads due to transient unbalance (due to loss of blade) and, on the other hand to identify the rotor/stator contact which leads to the failure of the blade. Thanks to the 
comprehension of the rotor dynamics, the scenario of the release of the blade can be identified or defined with a better confidence.

On the other hand, the impact of the blade on the stator part remains a main mechanical problem. By the past, the main subject was the dimensioning of the containment shield. Number of investigators have performed experimental studies and have created analytical models of the rotor burst and containment process (for example [5] and [6]). In order to reduce the time and cost of development of new engines, the Finite Element Method is used and allows to predict comparatively the ability of containment shield [7, 8]. Also Herran and al. [9] have conducted simulations in order to simulate blades impact and to define the required model for the fragmentation of the blade. In this work, blades profiles modelling with thick shells led to the assumption of no deformation in the thickness of the material and the impossibility of refinement of the profile. Even if a choice of volumic elements to model these blades seems more appropriate, however, we retain this blade modelling with thick shells mainly to avoid too fine meshes that would lead to important time calculations. This simplification was assumed to be acceptable. Thus, these simulations allow us to define a methodology for modelling the FE free turbine that ONERA has incorporated into a recent model of a helicopter engine.

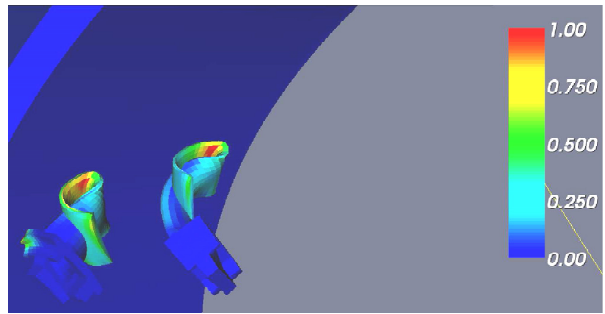

Figure 1: $\quad$ Plastic strain of two blades on a "blade shedding" shield [9].

In the present article, the work is focused on the modelling of the majority of the engine with volumic and shell elements and the resolution of the problem with the Newmark explicit algorithm implemented on the Europlexus software. The simulation is conducted for $10 \mathrm{~ms}$ to represent all of the highly nonlinear phenomenon.

\section{Blade shedding modelling}

\subsection{Dynamic non linear analysis}

The Europlexus code used in this paper is best adapted to rapid dynamic phenomena taking into account the geometric non linearity (large displacements, large rotations, large strains), and the non-linearity of materials (plasticity, viscoplasticity, etc).

Europlexus is an explicit dynamic software using a Newmark time integration scheme. At each time step, the calculation is defined as follows: 
- The $\mathrm{u}^{n}, \mathrm{v}^{n}, \mathrm{a}^{n}$ values are initially known (the time step is $t^{n}$ )

- Velocity calculation with a predictor scheme:

$$
\vec{v}^{n+\frac{1}{2}}=\vec{v}^{n}+\frac{\Delta t^{n}}{2} \cdot \vec{a}^{n}
$$

- Displacements calculation for the next time step:

$$
\vec{u}^{n+1}=\vec{u}^{n}+\Delta t^{n} \cdot \vec{v}^{n+\frac{1}{2}}
$$

- Internal efforts calculation using the behavior law,

- Acceleration calculation for the next time step:

$$
\vec{a}^{n+1}=\underline{\underline{m}}^{-1}\left(F_{e x t}^{n+1}-F_{i n t}^{n+1}\right)
$$

- Final velocities calculation:

$$
\vec{v}^{n+1}=\vec{v}^{n+\frac{1}{2}}+\frac{\Delta t^{n}}{2} \cdot \vec{a}^{n+1}
$$

The contact forces are obtained by the penalty method.

The major difficulty in this work is to obtain the most judicious compromise between the mesh size of our model and time step. Basically, dynamic fast time calculations are inversely proportional to the size of mesh. Then, we will focus mainly to conduct the full calculation for the first non-linear phase (around 10 $\mathrm{ms})$.

\subsection{FE methodology}

\subsubsection{Mesh rules}

The CRD unit of the DADS department has a great experience in computing and impact crash at high speed. The implementation methodology is to build a CAD model adapted to future needs as mesh size. In this context, the calculation of blade loss will be easier.

In order to correctly represent the bending stress of the volumic elements is necessary to discretize the thickness with at least three elements. So for weak thicknesses, elements might become too small resulting calculations too long for a simple parametric study. That is why the structure is mainly meshed with shell elements and that the bricks are used only for cases where the thickness are important, or to add the missing mass.

The screws were not represented thoroughly (real geometry). In our case, beam elements are used. They can model the mechanical links in terms of effort in tension and torsion (BSHT and BSHR EUROPLEXUS elements). These kind of beam elements are also used to model the shaft bearings. The rotor is linked with a beam element to the stator model.

Table 1: $\quad$ Mass and gravity center position ( $\mathrm{CAD} / \mathrm{FE}$ model).

\begin{tabular}{|c|c|c|c|c|}
\hline & ENGINE PARTS & F.E MODEL & CAD & $\%$ \\
\hline \multirow{2}{*}{ MASS (g) } & Part 1 (Fig.2) & 8900 & 9330 & -4.6 \\
\cline { 2 - 5 } & Part 2 (Fig.2) & $\mathbf{8 5 0 9}$ & $\mathbf{8 8 1 5}$ & -3.5 \\
\hline \multirow{2}{*}{$\begin{array}{c}\text { CG (Z, rotor axe) } \\
\text { (mm) }\end{array}$} & Part 1 & 1046 & 1047.5 & - \\
\cline { 2 - 5 } & Part 2 & 652.8 & 651.8 & - \\
\hline
\end{tabular}




\subsubsection{Mass and inertia analysis}

The next figures show two complexes engine parts and table 1 compares the real mass and gravity center position of the CAD with the model.
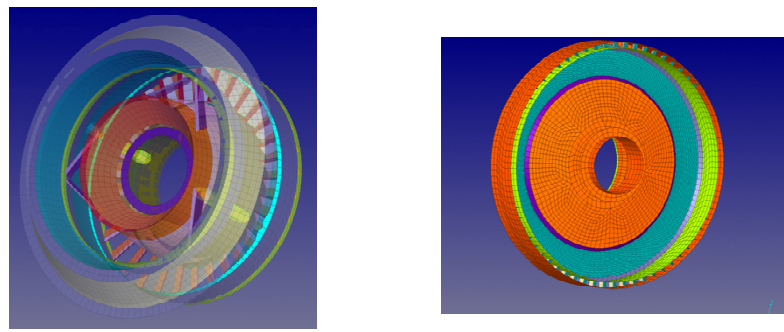

Figure 2: $\quad$ Example of two FE pieces of the complete engine.

The difference between the CAD complete model and the FE model leads to an acceptable difference lower than $5 \%$ respecting the mass, gravity center position and inertia moments.

\subsubsection{Engine mounting load methodology and calculation}

3.2.3.1 F.E model of the complete engine Figure 3 shows the complete model mesh used for the "blade-shedding" calculation.
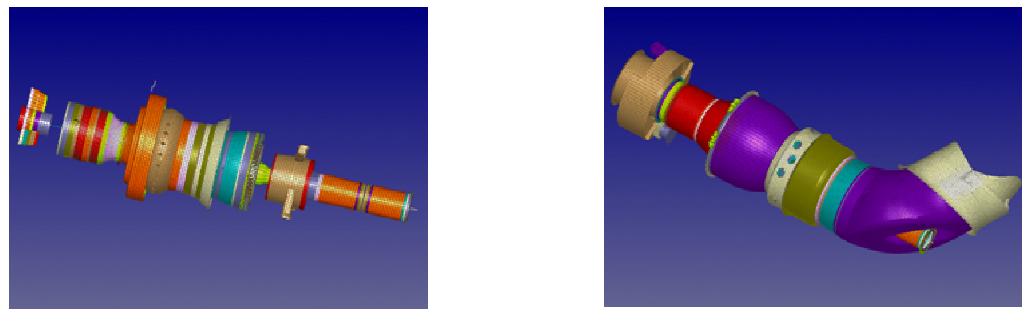

Figure 3: Complete FE model of the engine.

The complete F.E model contains:

- 254278 Nodes,

- 760 DST3 (3 nodes shell element),

- 83439 Q4GS (4 nodes shell elements),

- 91768 CUB8 (8 nodes hexaedric brick elements),

- 19584 SHB8 (8 nodes hexaedric thick shell elements for the blade profile),

- 10 BSHT (spring elements used specially for bearing),

- 388 POUT (Beam element).

3.2.3.2 Engine mountings loads modelling In this section a presentation of the method to calculate the mounting loads during the "blade shedding" phenomenon is exposed. The engine is linked to the helicopter by an isostatic mounting defined by two connecting rods at the front of the engine and a CV joint at the rear. Figure 4(a) shows the front mounting modelled with hexahedral elements and the rear linking tube meshed with shell elements. 

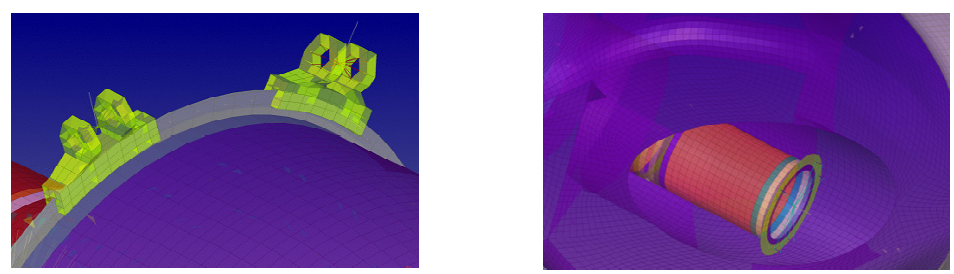

Figure 4: $\quad$ Front (a) and rear (b) mountings FE modelling.

Then a cinematic relationship rigid element defines a "master" node at each mounting. This "master" node represents the average position of the mounting (Figure 4(b)).

A beam element is connected to one end to this "master" node, the other end to the node of helicopter mounting. In the case of an engine mounting, this node is clamped and so the helicopter loads are overestimated. For the rear mounting, the $\mathrm{CV}$ joint is realized by a user beam element (BSHT element) which allows the freedom of rotation and provides the forces for this mounting. After that the bending moment on the mounting is deduced.

3.2.3.3 Blades-loss method: "blade shedding" For the blades-loss and complete FE simulation, the methodology is given below:

- Do first prestressing calculation (shaft screw) with damping,
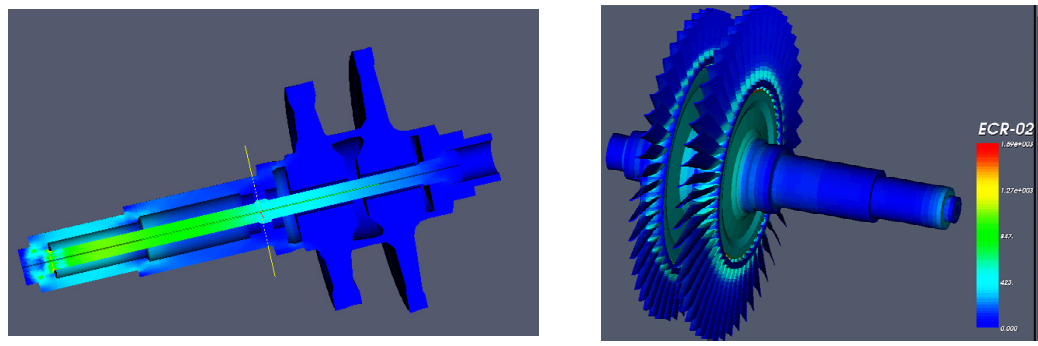

Figure 5: $\quad$ Prestressing calculation and centrifugal forces calculation.

Indeed, to ensure the cohesion of the rotating parts of the rotor, a pretension stress is carried out by a tie-bolt. The tie-bolt is set during the assembly of the shaft and ensures the cohesion between the disks and the shaft. The tie-bolt is put in tension with a screw (Figure 5). The remaining elastic force of the tie-bolt permits a perfect disk-shaft cohesion. This assembly is essential because if the tie-bolt were to break, there would be a separation of the rotating parts. Therefore, the shaft is modelled with volumic elements to achieve correctly the prestressing method and to take into account the gyroscopic effects. It is important to note that, using a dynamic code with explicit algorithm for an axial prestressing calculation (with a continuous mesh) constitutes a unique and original method. This method can detect the opening of the coupling (no tensions 
on the border element), the plastification of the rotor and takes into account all the effects on the rotor dynamics of the free turbine.

- Restart with displacements, stresses, hardening parameters of the first prestressing calculation,

- Apply centrifugal forces with damping on all structure,

A calculation to verify the stability of our model is achieved before starting the calculations of rotor rotation and centrifugal forces (Figure 5).

- Apply rotor initial velocity,

The same restart method used for the stabilisation calculation is achieved. Thus, the calculation restarts with the final conditions of the previous calculation. These conditions are now the initial conditions used to simulate the blade-loss and "blade shedding" phenomenon (Fig. 6).

- Perform a "Blade shedding" calculation with a Turbomeca released blade scenario.
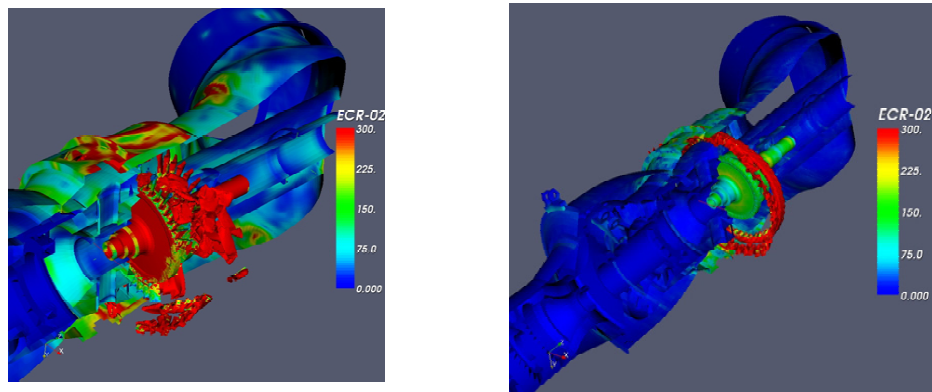

Figure 6: Sequential and simultaneous blades-loss calculation.
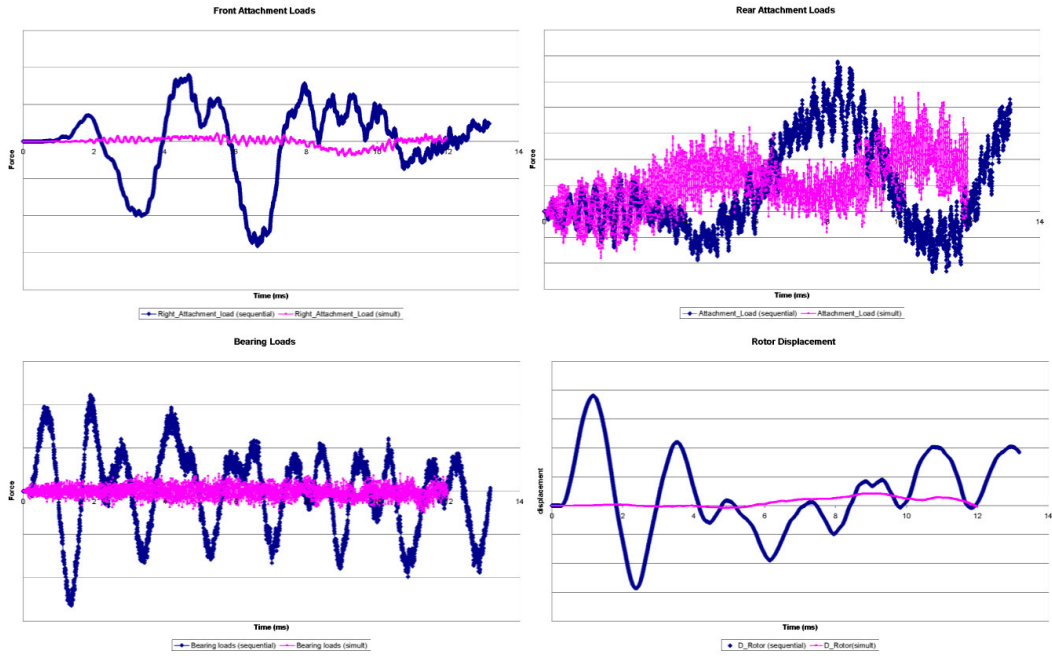

Figure 7: Engine mounting, bearing loads and rotor displacement (sequential and simultaneous blades-off). 
A simultaneous blade-loss calculation is performed to compare and observe the significant bearings efforts in the case of a non symmetrical blades-off (Figure 6).

\subsection{Engine mounting efforts}

The transient response of the engine during the blade shedding has been numerically analysed. The efforts in the engine mounting components have been especially post treated. The calculation has been obtained for 10 to $13 \mathrm{~ms}$ which is relevant compared with the sequential blades-off of $5 \mathrm{~ms}$ only. Figure 7 shows the different loads calculated in the shaft bearing and bolted assemblies. We can observe an important unbalance. A first approach compared with measurement has shown an acceptable correlation. The ratio between the sequential blades-off and simultaneous case is close to 10 for the front engine mountings and only 1.3 for the rear linking. Respect to the shaft bearing, this ration is 5 .

The rotor displacement can be observed in figure 7. Here, the same comparison with a simultaneous blade-off is done. The displacement of the rotor with a sequential blade-loss sequence is significant, higher than in the simultaneous case (ratio close to 9). During this first non linear phase, the rotor loads are introduced by the transient unbalance due to the sequential blades-off.

\section{Conclusion}

The complete methodology to simulate the blade shedding phenomenon has been achieved in this work, using the dynamic explicit code Europlexus. This particular mechanical protection naturally coupled with the classical electronic protection is used in case of overspeed. Furthermore, the simulation was conducted taking into account the shaft assembly, taking care to simulate the prestressing due to the tie-bolt. A comparison between two simulations was performed, one reporting a sequential blades-off and the other simulating a simultaneous loss of the blades. Even if the second simulation has very little interest, it helps us to observe the significant structure loads for a significant unbalance. The work presented in this paper allows us to analyse the dynamic response of a turboshaft engine during blade shedding. Finally, the rotor unbalance load depends of the blade-off sequence. A FE model using an Explicit Newmark time algorithm, allows one to observe with the first non linear phase. Recently, Turbomeca uses simplify model to analyse high level of vibration up to $200 \mathrm{~ms}$ taking into account gyroscopic effect [3]. All these theoretical results obtained in this work require a comparison with tests in a future work.

\section{Acknowledgements}

The authors gratefully acknowledge Turbomeca for its support. This work takes place in the framework of the MAIA mechanical research and technology program sponsored by CNRS, ONERA and SAFRAN Group. 


\section{References}

[1] Sun, G., Palazzolob, A., Provenzac, A., Lawrencec, C., Carney, K., Long duration blade loss simulations including thermal growths for dual-rotor gas turbine engine. Journal of Sound and Vibration, 316, pp. 147-163, 2008.

[2] Guilhen, P.M., Berthier, P., Ferraris, Guy, Lalanne, M., Instability and unbalance response of dissymetric rotor bearing systems, ASME J. Vibr. Ac. Str. Rel. Des., 110 July 1988.

[3] Herran, M., Rapport annuel de thèse : Prédiction des efforts aux attaches lors d'un blade shedding, Tech. Rep., Turbomeca, 2008.

[4] Herran, M., Nélias, D., Ortiz, R., Draft : implementation of rotor dynamics effects into the europlexus code fort the prediction of transient dynamic loading of engine mountings due to blade shedding unbalance, Proceedings of TURBOEXPO 2009, Proceedings of ASME Turbo Expo 2009: Power for Land, Sea and Air, June 8-12, 2009, Orlando, USA.

[5] Mangano, G.J., Studies of engine rotor fragment impact on protective structures, Agard Con \& Proc., Impact Damage Tolerance of Structure, 41st Meeting of the Structures and Materials panel, 186, 1975.

[6] McCarthy, D., Types of rotor failure and characteristics of fragments, $A$ workshop held at the Massachusetts, Institute of Technology, NASA CP2017, pp. 65-92, 1977.

[7] Kelvin, Y. Ng., Turbine rotor burst containment analysis using MSC/Dytran, an analytical approach to predicting primary containment, MSC 1996 World Users' Conference Proceedings.

[8] Kraus, A., Frischbier, J., Containment and penetration simulation in case of blade loss in a low pressure turbine, Proceedings of the DYNAmore LSDYNA Forum 2002 19-20 September, Bad Mergentheim (2002).

[9] Herran, M., Chalons, H., Nélias, D., Ortiz, R., Modélisation de l'impact d'une pale contre un blindage lors du "blade Shedding", Colloque Vibrations Chocs et bruit, Lyon (France), 2008. 